\title{
Intracranial Gliomas: Association between MRI Features and Tumor Grade
}

\author{
Islam $\mathbf{M R}^{1}$, Islam $\mathrm{KMT}^{2}$, Rahman $\mathbf{M}^{3}$, Hossain $\mathbf{M}^{4}$, Haque $\mathbf{R}^{5}$, HosainATMM ${ }^{6}$
}

\begin{abstract}
:
Purpose: Intracranial gliomas are not uncommon in our country. The therapeutic management and prognosis in patients with gliomas depend on the reliable distinction between high and low-grade gliomas. The purpose of the present study was to determine whether any association exists between various individual MR imaging features and histopathologically determined tumor grade of intracranial gliomas. Our target was to explore whether MRI could enable correct determination the grading of gliomas which will help early diagnosis, staging, grade assessing, determining the appropriate treatment, preoperative planning for surgical resection and post operative management plan.

Materials and Methods: It was a cross sectional type of observational study. This study was conducted on 44 cases of intracranial gliomas. Only patients having histopathologically proven intracranial gliomas who underwent surgery or stereotactic biopsy were considered as a study population.

Results: The present study revealed that there is significant association between the MR imaging features and histopathological grading of intracranial gliomas. Contrast enhancement in MRI showed most significant $(p<0.001)$. Other significant MR imaging features were peritumoral edema $(p=0.001)$, tumor border definition ( $p=0.001)$, necrosis or cystic change of tumor $(p=0.001)$ and mass effect $(p=0.003)$. In this study preoperative MRI diagnosis was correct in 39 patients with accuracy of $88.6 \%$. Sensitivity, specificity, positive predictive value and negative predictive value of MRI in detecting tumor grade were $86.4 \%, 90.9 \%, 90.5 \%$ and $87 \%$ respectively.

Conclusion: MRI is sensitive, specific and accurate in assessing the grade of gliomas. Contrast enhancement is the most important predictor. Peritumoral edema, tumor border definition, mass effect and necrosis/cystic changes of tumor are also important indicators of tumor grade.
\end{abstract}

Key words: MRI (magnetic Resonance Imaging), Gliomas, Astrocytoma, Glioblastoma.

Bang. J Neurosurgery 2019; 8(2):93-99

\section{Introduction}

Gliomas account for approximately $40 \%$ to $67 \%$ of primary brain tumors. The incidence rate is

1. Dr. Md. Rokibul Islam, Medical Officer, Department of Neurosurgery, Bangabandhu Sheikh Mujib Medical University, Dhaka

2. Dr. KM Tarikul Islam, Associate Professor, Department of Neurosurgery, Bangabandhu Sheikh Mujib Medical University, Dhaka

3. Dr. Moshiur Rahman, Assistant professor, Department of Neurosurgery, Holy Family Red Crescent Medical College \& Hospital, Dhaka

4. Dr. Mohammad Hossain, Associate Professor, Department of Neurosurgery, Bangabandhu Sheikh Mujib Medical University, Dhaka

5. Dr. Raziul Haque, Professor, Department of Neurosurgery, Dhaka Medical College \& Hospital.

6. Prof. ATM Mosharef Hosain, Professor \& Chairman, Department of Neurosurgery, Bangabandhu Sheikh Mujib Medical University, Dhaka

Address of Correspondence: Dr. Md. Rokibul Islam, Medical officer, Department of Neurosurgery, Bangabandhu Sheikh Mujib Medical University, Dhaka. email: mail2rokib@gmail.com Tel: 01711452526 approximately 4.0 per 100,000 populations in males and just slight lower in females ${ }^{1}$. Two thirds of all brain tumors are primary neoplasms. Approximately half of all primary brain tumors are glial neoplasm and more than three quarter of all gliomas are astrocytomas. More than three quarters of all astrocytomas are anaplastic astrocytomas and glioblastomamultiforme. Approximately 16500 new cases of primary brain tumors are diagnosed yearly in the USA. Gliomas are the most common of them and comprise $1.35 \%$ of all cancers in the USA ${ }^{2}$. The grading of gliomas is required for determining prognosis and directing clinical management ${ }^{3}$.

Two major limitations are associated with histopathologic grading of gliomas: inherent sampling error associated with stereotactic biopsy and inability to evaluate residual tumor tissue after cytoreductive surgery. Malignant gliomas are known to infiltrate the parenchyma following vascular channels of the white 
matter tracts. This may not be readily appreciated if there is no signal intensity abnormality or enhancement on conventional MR images. Hence, histopathologic grading of gliomas has disadvantages and intrinsic error. The advantage of state- of-the-art MR imaging techniques in evaluating cerebral gliomas is the ability to sample not only the entire lesion, but also the adjacent brain tissue for physiologic and metabolite alterations ${ }^{4}$.

By using primarily morphologic imaging modalities, such as MR imaging, a close correlation has been found between intense gadopentetatedimeglumine enhancement of cerebral astrocytomas and histologic areas of malignant neovascularity and endothelial proliferation. Therefore, contrast enhancement is associated with a higher grade of malignancy ${ }^{5}$.

Histological grading is a means of predicting the biological behaviour of a neoplasm. In the clinical setting, tumor grade is a key factor influencing the choice of therapies, particularly determining the use of adjuvant radiation and specific chemotherapy protocols. The WHO classification of tumors of the nervous system includes a grading scheme that is a malignancy scale ranging across a wide variety of neoplasms rather than a strict histological grading system ${ }^{6}$.

\section{Method \& Materials:}

It was a cross sectional type of observational study. This study was carried out at Department of Neurosurgery, Dhaka Medical College Hospital $(\mathrm{DMCH})$, Dhaka, Bangladesh during the period of July 2013 to May 2015. Purposive sampling according to availability of the patients and strictly considering the inclusions and exclusions criteria. Only patients having histopathologically proven intracranial gliomas who underwent surgery or stereotactic biopsy were considered as a study population. MRI features that are taken for study are location of tumor, contrast enhancement, peritumoral edema, tumor border definition, mass effect of tumor\&Necrosis/cystic changes of tumor.For the validity test sensitivity, specificity, accuracy, positive predictive value and negative predictive value of MRI in detecting the grade of intracranial gliomas were calculated after confirmation of the diagnosis by histopathology. Data was collected from Department of Neurosurgery, Dhaka Medical College Hospital (DMCH), Bangabandhu Sheikh Mujib Medical University (BSMMU), and some private hospitals of Dhaka city.

\section{Results:}

This study was conducted on 44 cases of intracranial gliomas at Department of Neurosurgery, Dhaka Medical
College Hospital, Dhaka. The purpose of this study was to find the association between MR imaging features and tumor grade of intracranial gliomas. In present study the age of the patients ranged from 4 years to 65years. Mean age was 36.3 years. The highest incidence was in between 31- 40 years.

Age incidence in intracranial gliomas is an important variable, which is observed to vary from study to study. In present study, no particular age was found susceptible to develop gliomas. Variation of gliomas with respect to sex is not also uncommon. The present study showed a male predominance $(63.6 \%)$ at the ratio of 1.75:1 bearing consistency with findings of other investigators ${ }^{7}$.

In this study, out of 44 patients of intracranial gliomas, regarding histopathological subtypes of tumor, $43.2 \%$ patients had glioblastoma, $31.8 \%$ had diffuse astrocytoma, 9.1\% had pilocytic astrocytoma, $6.8 \%$ had anaplastic astrocytoma, $6.8 \%$ had ependymoma and $2.3 \%$ had oligodendroglioma.

In this study regarding histopathological grading, $9.1 \%$ was grade I gliomas, $40.9 \%$ was grade II gliomas, $6.8 \%$ was grade III gliomas and $43.2 \%$ were grade IV gliomas.
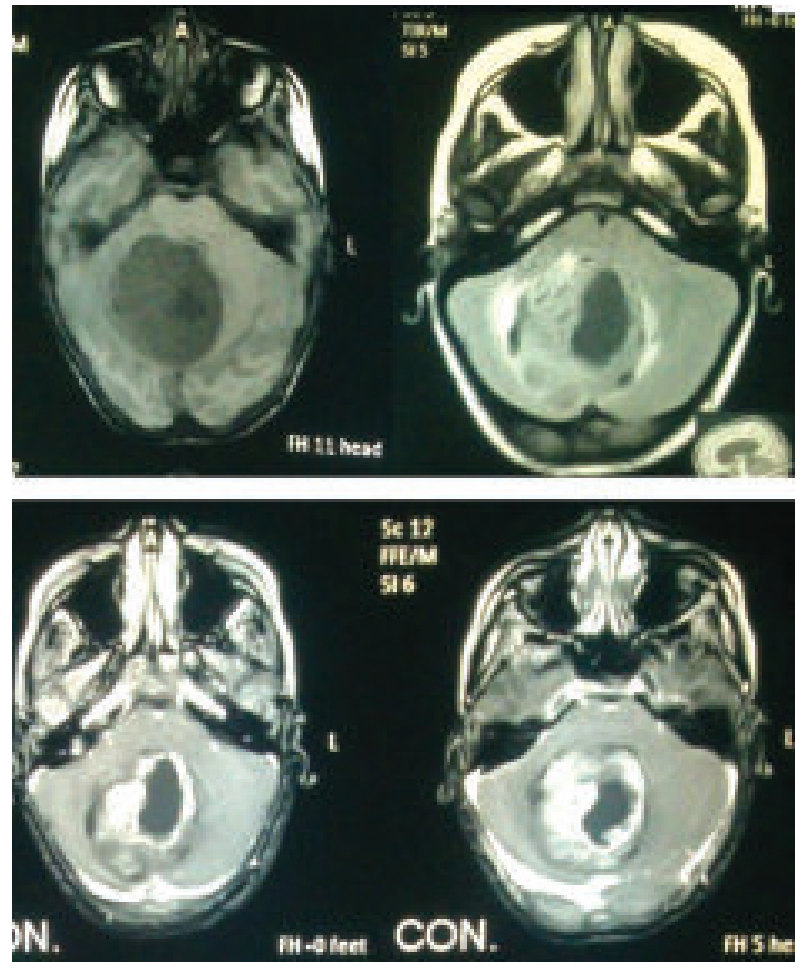

Fig.-1: MRI of WHO Grade I gliomas (Pilocytic Astrocytoma): T1WI, FLAIR \& T1 Contrast images showing hypointense cystic cerebeller lesion with mass effect \& mild peritumoral edema, enhanced with contrast. 

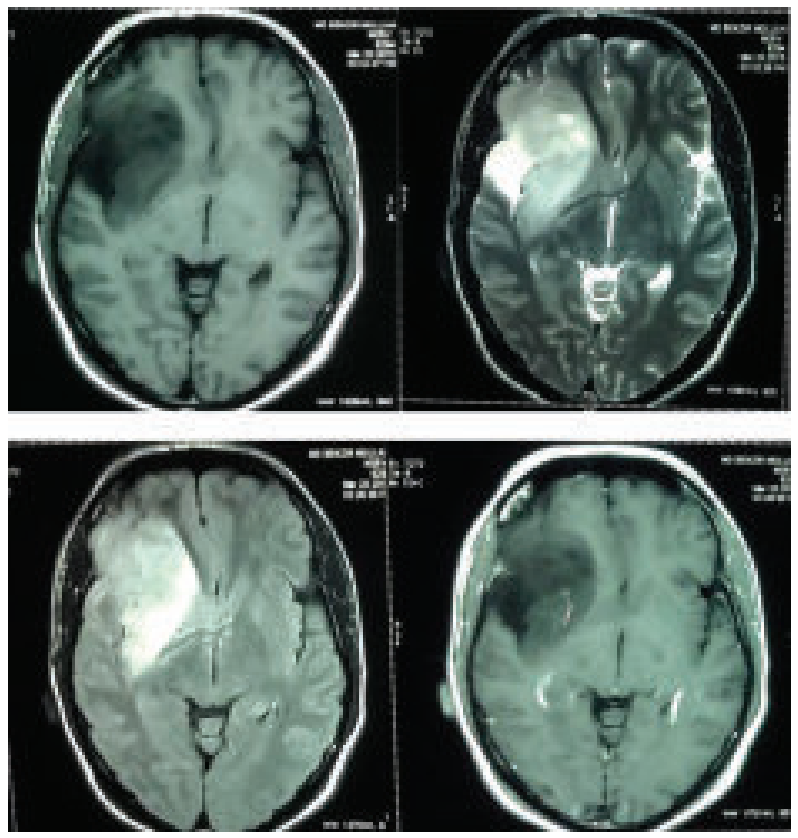

Fig.-2: MRI of WHO Grade II gliomas (Diffuse Astrocytoma): T1WI, T2WI, FLAIR \& T1 Contrast images showing hypointense right insular (hemispheric) lesion with mass effect \&peritumoral edema, not enhanced with contrast and there is no necrosis/cystic changes.
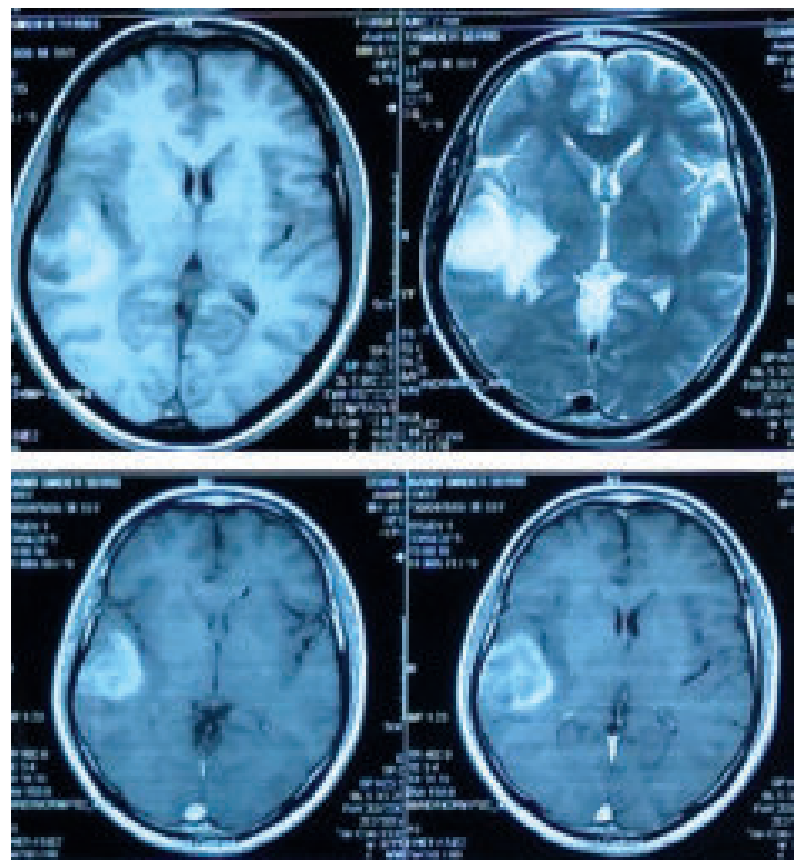

Fig.-3: MRI of WHO Grade III gliomas (Anaplastic Astrocytoma): showing right temporal (hemispheric) well defined hypointense lesion on T1WI, which is heterogeneously hyperintense on T2WI, with moderate peritumoral edema and moderately heterogenous enhancement with contrast is noted.
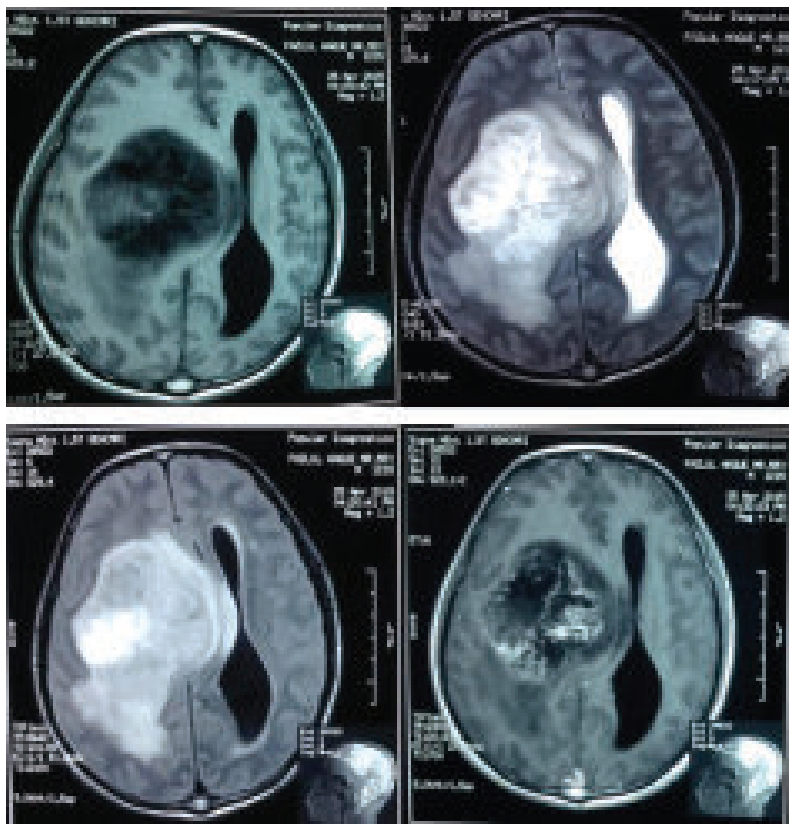

Fig.-4: MRI of WHO Grade IV gliomas (Glioblastoma): T1WI, T2WI, FLAIR \& T1 Contrast images showing poorly circumscribed right parietal (hemispheric) lesion with marked mass effect \& marked peritumoral edema, enhanced with contrast with presence of necrosis and cystic changes.

In the present study out of 44 patients, regarding contrast enhancement, $12(27.3 \%)$ patients showed no enhancement, $13(29.5 \%)$ patients showed mild to moderate enhancement and $19(43.2 \%)$ patients showed marked enhancement after administration of IV gadolinium contrast.

Table I

Distribution of patients by age $(n=44)$

\begin{tabular}{lcc}
\hline $\begin{array}{l}\text { Age group } \\
\text { in year }\end{array}$ & Frequency $(\mathrm{n})$ & Percentage (\%) \\
\hline$\leq 10$ & 3 & 6.8 \\
$11-20$ & 3 & 6.8 \\
$21-30$ & 11 & 25 \\
$31-40$ & 12 & 27.3 \\
$41-50$ & 5 & 11.5 \\
$51-60$ & 6 & 13.6 \\
$>60$ & 4 & 9.1 \\
\hline Total & 44 & 100 \\
\hline
\end{tabular}


In this study, out of 44 patients of gliomas 28 (63.6\%) were males and $16(36.4 \%)$ were females. The male to female ratio was $1.75: 1$.

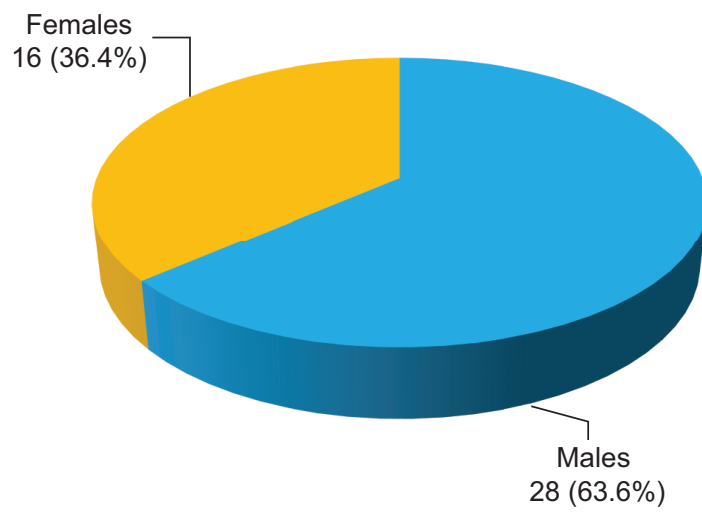

Fig.5: Pie-chart showing distribution of patients by $\operatorname{sex}(n=44)$.

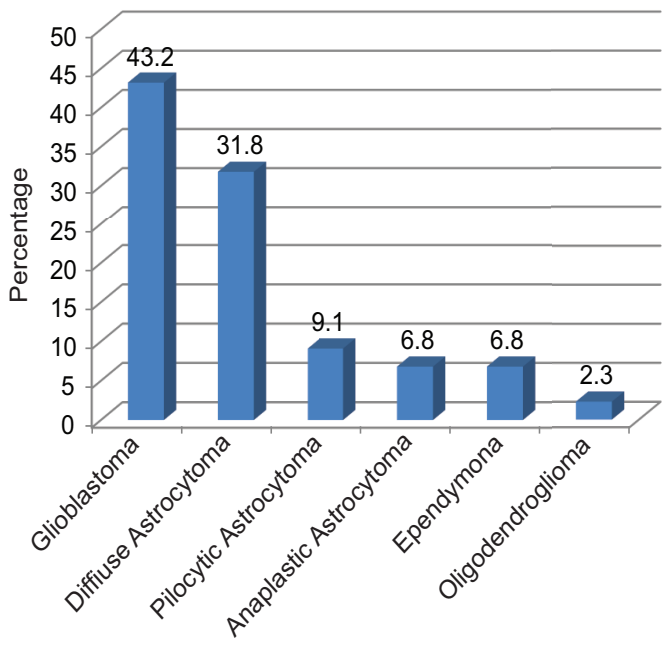

Fig.-6: Bar diagram showing distribution of patients by histopathological subtype of gliomas $(n=44)$.

Table-II

Distribution of patients by WHO grading of gliomas by histopathology $(n=44)$

\begin{tabular}{lcc}
\hline WHO grading & Frequency $(\mathrm{n})$ & Percentage (\%) \\
\hline Grade I & 4 & 9.1 \\
Grade II & 18 & 40.9 \\
Grade III & 3 & 6.8 \\
Grade IV & 19 & 43.2 \\
\hline Total & 44 & 100.0 \\
\hline
\end{tabular}

Table-III

Association between contrast enhancement and WHO grading of gliomas $(n=44)$

\begin{tabular}{lcccc}
\hline WHO grade & No & $\begin{array}{c}\text { Contrast enhancement } \\
\text { Mild to moderate } \\
\text { enhancementn (\%) }\end{array}$ & $\begin{array}{c}\text { Marked } \\
\text { enhancementn (\%) }\end{array}$ \\
\hline Grade I & $2(16.7)$ & $2(15.4)$ & $0(0.0)$ & $4(9.1)$ \\
Grade II & $10(83.3)$ & $6(46.2)$ & $2(10.5)$ & $18(40.9)$ \\
Grade III & $0(0.0)$ & $1(7.7)$ & $2(10.5)$ & $3(6.8)$ \\
Grade IV & $0(0.0)$ & $4(30.8)$ & $15(79.0)$ & $19(43.2)$ \\
\hline Total & $12(100.0)$ & $13(100.0)$ & $19(100.0)$ & $44(100.0)$ \\
\hline
\end{tabular}

Table-IV

Association between Peritumoral edema and WHO grading of gliomas ( $n=44)$

\begin{tabular}{lcccc}
\hline WHO grade & \multicolumn{3}{c}{ Total } \\
& $\begin{array}{c}\text { Peritumoral edema } \\
\text { edeman (\%) }\end{array}$ & $\begin{array}{c}\text { Mild to } \\
\text { moderate edeman }(\%)\end{array}$ & $\begin{array}{c}\text { Marked } \\
\text { edeman }(\%)\end{array}$ \\
\hline Grade I & $3(37.5)$ & $1(5.0)$ & $0(0.0)$ & $4(9.1)$ \\
Grade II & $5(62.5)$ & $13(65.0)$ & $0(0.0)$ & $18(40.9)$ \\
Grade III & $0(0.0)$ & $3(15.0)$ & $0(0.0)$ & $3(6.8)$ \\
Grade IV & $0(0.0)$ & $3(15.0)$ & $16(100.0)$ & $19(43.2)$ \\
\hline Total & $8(100.0)$ & $20(100.0)$ & $16(100.0)$ & $44(100.0)$ \\
\hline
\end{tabular}


Table-V

Association between tumor border definition \& WHO grading of gliomas $(n=44)$

\begin{tabular}{lccc}
\hline WHO Grade & \multicolumn{2}{c}{ Tumor border definition } & p value \\
\cline { 2 - 3 } & Well circumscribed $\mathrm{n}(\%)$ & Poorly circumscribedn $(\%)$ & \\
\hline Grade I & $4(16.7)$ & $0(0.0)$ & 0.001 \\
Grade II & $15(62.5)$ & $3(15.0)$ & \\
Grade III & $1(4.2)$ & $2(10.0)$ & \\
Grade IV & $4(16.7)$ & $15(75.0)$ & \\
\hline Total & $24(100.0)$ & $20(100.0)$ & \\
\hline
\end{tabular}

Table-VI

Association between mass effect \& WHO grading of gliomas $(n=44)$

\begin{tabular}{lcccc}
\hline WHO Grade & \multicolumn{3}{c}{ Mass effect } & p value \\
\cline { 2 - 4 } & $\begin{array}{c}\text { No mass } \\
\text { effectn }(\%)\end{array}$ & $\begin{array}{c}\text { Mild to moderate mass } \\
\text { effectn }(\%)\end{array}$ & $\begin{array}{c}\text { Marked mass } \\
\text { effectn }(\%)\end{array}$ & \\
\hline Grade I & $0(0.0)$ & $4(13.8)$ & $0(0.0)$ & 0.003 \\
Grade II & $1(33.3)$ & $17(58.6)$ & $0(0.0)$ & \\
Grade III & $0(0.0)$ & $2(6.9)$ & $1(8.3)$ & \\
Grade IV & $2(66.7)$ & $6(20.7)$ & $11(91.7)$ & \\
\hline Total & $3(100.0)$ & $29(100.0)$ & $12(100.0)$ & \\
\hline
\end{tabular}

Table-VII

Association between the Necrosis/Cystic change \& the WHO grading of gliomas

\begin{tabular}{lccc}
\hline WHO grade & \multicolumn{2}{c}{ Necrosis/ Cystic change of tumor } & p value \\
\cline { 2 - 3 } & Presentn (\%) & Absentn $(\%)$ & \\
\hline Grade I & $0(0.0)$ & $4(13.3)$ & 0.001 \\
Grade II & $0(0.0)$ & $18(60.0)$ & \\
Grade III & $0(0.0)$ & $3(10.0)$ & \\
Grade IV & $14(100.0)$ & $5(16.7)$ & \\
\hline Total & $14(100.0)$ & $30(100.0)$ & \\
\hline
\end{tabular}

Table-VIII

Comparison between histopathology diagnosis and MRI diagnosis of gliomas

\begin{tabular}{lccc}
\hline MRI diagnosis & \multicolumn{2}{l}{ Histopathological diagnosis $($ Gold standard test) } & Totaln (\%) \\
\cline { 2 - 3 } & $\begin{array}{c}\text { Low grade } \\
\text { (grade I \& II)n (\%) }\end{array}$ & $\begin{array}{c}\text { High grade } \\
\text { (grade III \& VI)n (\%) }\end{array}$ & \\
\hline Low grade & $19(86.4)$ & $2(9.1)$ & $21(47.7)$ \\
High grade & $3(13.6)$ & $20(90.9)$ & $23(52.3)$ \\
\hline Total & $22(100.0)$ & $22(100.0)$ & $44(100.0)$ \\
\hline
\end{tabular}


Table-IX

Validity test of MRI in detecting tumor grade of intracranial gliomas

\begin{tabular}{lccc}
\hline Validity test & Estimated Value (\%) & \multicolumn{2}{c}{$95 \%$ confidence interval } \\
\cline { 3 - 4 } & & Lower Limit & Upper Limit \\
\hline Sensitivity & 86.4 & 71.1 & 93.6 \\
Specificity & 90.9 & 75.6 & 98.1 \\
Positive predicative value & 90.5 & 74.4 & 98.0 \\
Negative predicative value & 87.0 & 72.3 & 93.9 \\
Accuracy & 88.6 & 73.3 & 95.9 \\
\hline
\end{tabular}

\section{Discussion:}

The grading of gliomas is required for determining prognosis and directing clinical management3. Histological grading is a means of predicting the biological behavior of a neoplasm. In the clinical setting, tumor grade is a key factor influencing the choice of therapies, particularly determining the use of adjuvant radiation and specific chemotherapy protocols 6

The therapeutic management and prognosis in patients with gliomas depend on the reliable distinction between high and low-grade gliomas. Imaging in patients with brain tumors aims toward the determination of the localization, extend, type, and malignancy of the tumor. Imaging is being used for primary diagnosis, planning of treatment including placement of stereotaxic biopsy, resection, radiation, guided application of experimental therapeutics, and delineation of tumor from functionally important neuronal tissue8. CT and MRI are the mainstays of imaging in current practice. MR imaging with its multiplanar capabilities and superior contrast resolution is now modality of choice9.

The present study showed significant association between the contrast enhancement and WHO grading of gliomas $(p=<0.001)$ bearing consistency with findings of other investigators 5,10 . There is direct relationship between contrast enhancement and neovascularity present in high grade tumors 9 . In lowgrade gliomas with little or no alteration of BBB, contrast agent did not enter the extravascular space and no significant contrast enhancement was observed10.

In this study regarding presence of peritumoral edema, in $8(18.2 \%)$ patients there is no edema, $20(45.5 \%)$ patients showed mild to moderate edema and $16(36.4 \%)$ patients showed presence of marked edema. Some investigators demonstrated direct correlation between peritumoral edema with tumor grade $(p<0.01) 13$. Other authorsfound significant association between the perilesional edema and pathological grade of astrocyticgliomas10. Present study also demonstrated significant association $(p<0.001)$ between peritumoral edema and WHO grade of intracranial gliomas.

In present study $54.5 \%$ was well circumscribed lesion and $45.5 \%$ was poorly circumscribed lesion. Other study10 found significant association between definition of tumor border and the pathological grade of astrocyticgliomas $(p<0.02)$. Our study demonstrated significant association between tumor border definition \& WHO grading of gliomas $(p=0.001)$

In this study $29(65.9 \%)$ of patients showed mild to moderate mass effect followed by $12(27.3 \%)$ marked mass effect and 3(6.8\%) no mass effect. This showed significant association between the mass effect and the WHO grading of gliomas $(p=0.003)$. For gliomasmass effect was the best predictor for assessing tumor grade $(p=.0000)$ using MR imaging3. There is significant association between the mass effect and pathological grade of astrocyticgliomas $(p<0.001) 10$

In this study necrosis or cystic change was absent in $68.2 \%$ and present in $31.8 \%$. It is noted that this $31.8 \%$ were grade IV lesions. Our study also revealed significant association between necrosis/cystic change of tumor with tumor grade $(p=0.001)$. This was also found in other study 3,10

In present study, low grade gliomas characteristically were well defined; displayed no or little mass effect, peritumoral edema; nonenhancing or mild to moderate enhancing lesion on IV contrast administration; and were without necrosis/cystic changes. High gradegliomas were less well defined of poorly defined border; exhibited more mass effect, vesogenic 
edema; significant enhancing lesions; and majority presented with necrosis/cystic changes.

In this study preoperative MRI diagnosis was correct in 39 patients with accuracy of $88.6 \%$. One study showed the overall accuracy rate of MR imaging was $83.3 \%$ for observer 1 and $80.6 \%$ for observer 2 for grading of astrocytic-series gliomas 3 . Preoperative MRI diagnosis was correct in 50 patients with accuracy of $94 \% 13$

In present study out of all patients, 21 were diagnosed as low grade gliomas by MRI, among them 19 cases were true positive 02 cases were diagnosed as low grade gliomas by MRI but was diagnosed as high grade gliomas by histopathology. These 2 cases were false positive. Out of all cases of gliomas 23 were diagnosed as high grade gliomas by MRI, among them 20 cases were true positive and 03 cases were diagnosed as high grade gliomas by MRI but were diagnosed as low grade gliomas by histopathology. These 3 cases were false positive. Sensitivity, specificity, positive predictive value and negative predictive value of MRI in detecting tumor grade were $86.4 \%, 90.9 \%, 90.5 \%$ and $87 \%$.

\section{Conclusion}

The present study revealed that there is significant association between the MR imaging features and histopathological grading of intracranial gliomas. MRI is sensitive, specific and accurate in assessing the grade of gliomas. Contrast enhancement is the most important predictor. Peritumoral edema, tumor border definition, mass effect and necrosis/cystic changes of tumor are also important indicators of tumor grade.

\section{Recommendations}

Any patient suspected of having intracranial gliomas should undergo MR imaging with contrast studies to come to a conclusive preoperative diagnosis and to provide better management.

\section{References:}

1. Doran, SE \&Thorell, WE 2004, Brain tumors: populationbased epidemiology, environmental risk factors, and genetic and hereditary syndromes, in Youman's Neurological Surgery,
Winn, HR (ed.), $5^{\text {th }}$ edn, Saunders, USA, vol.1, pp. 807-816.

2. Sloan, A.E., Abdolvahvi, R. \&Hlatky, R. 2005. Gliomas, in Principles of Neurosurgery, Rengachary, S.S. \&Ellenbogen, R.G. eds., 2005. USA: Elsevier, pp.451-477.

3. Dean, B.L., Drayer, B.P., Bird, C.G., Flom, R.A., Coons, S.W. \& Carry, R.J., 1990. Gliomas: Classification with MR Imaging. Radiology, 174, pp.411-415.

4. Law., M., Yang., S., Wang, H., Babb, J.S., Cha, S., Knopp, E.A. \&Zagzag, D., 2003. Glioma grading: sensitivity, specificity, and predictive values of perfusion MR imaging and proton MR spectroscopic imaging compared with conventional MR imaging. American Journal of Neuroradiology, 24, pp.1989-1998.

5. Reimann, B., Papke, K., Hoess, N., Kuwert, T., Weckesser, M., Matheja, P., Wassmann, H., Heindel, W. \&Schober, O., 2002. Noninvasive grading of untreated gliomas: A comparative study of MR imaging and 3-(iodine 123)-L- \pm Methyltyrosine SPECT. Radiology, 225, pp.567-574.

6. Louis, D.N., Cavenee, W.K., Burger, P.C., Kleihues, P., et al., 2007. The $2007 \mathrm{WHO}$ Classification of Tumours of the Central Nervous System. ActaNeuropathol, 114, pp.97-109.

7. Taheri SM, Samadian M, Aghaei M, Jalali A, Shakiba M 2008, Evaluation of CT scan and MRI findings of pathologically proved gliomas in an Iranian population, Iranian Journal of Cancer Prevention. 4(1): pp 179-182

8. Jacobs, A.H., Kracht, L.W., Gossmann, A., Ruger, A.M. et al 2005. Imaging in Neurooncology. NeuroRx. 2, pp.333-347.

9. Knopp, E.A., Cha, S., Johnson, G., Majumdar, A., Golfinos, J.G., Zagzag, D., Miller, D.C., Kelly, P.J. \&Kricheff, II 1999. Glial Neoplasms: Dynamic Contrast-enhanced T2*- weighted MR imaging. Neuroradiology, 211, pp.791-798.

10. Watanabe, M., Tanaka, R. \& Takeda, N., 1992. Magnetic resonance imaging and histopahology of cerebral gliomas. Neuroradiology, 34, pp.463-469.

11. Asari, S., Makabe. T., Katayama, S., Itoh, T., Tsuchida, S. \&Ohmoto, T., 1994. Assessment of the pathological grade of astrocyticgliomas using an MRI score. Neuroradiology, 36, pp.308-310

12. Tervonen, O., Forbes, G., Scheithaurer, B.W. \& Dietz, M.J., 1992. Diffuse fibrillaryastrocytomas: correlation of MRI features with histopathologic parameters and tumor grade. Neuroradiology, 34, pp.173-178.

13. Chishty, I.A., Rafique, M.Z., Hussain, M., Akhtar, W., Ahmed, M.N., Sajjad, Z. \& Ali, S.Z., 2010. MRI Characterization and Histopathological Correlation of Primary Intra-axial Brain Glioma. Journal of Liaquat University of Medical and Health Sciences (JLUMHS) 09(02), pp.64-69. 